\title{
A Benchmark of Dynamic Reliability Methods for Probabilistic Safety Assessment
}

\author{
Xiaolei Pan \\ College of Automation Engineering, \\ Shanghai University of Electric Power, \\ Shanghai, China \\ e-mail: xiaolei.pan@shiep.edu.cn
}

\author{
Francesco Di Maio, Enrico Zio \\ Energy Department, \\ Politecnico di Milano, \\ Milano, Italy \\ e-mail: francesco.dimaio@polimi.it, enrico.zio@polimi.it
}

\begin{abstract}
Various dynamic reliability methods have been developed during the last several decades, such as Monte Carlo (MC) simulation, Dynamic Event Tree (DET) and Monte Carlo Dynamic Event Tree (MCDET) methods, to name a few. This paper benchmarks these particular methods by applying them to a classic level control dynamic system and to a realistic Emergency and Standby Power System (ESPS). The analysis is done with respect to different component aging dynamics and transition rate state dependences. Efficiency and computational cost are evaluated. The results show that: (1) the dynamic methods indeed are capable of capturing the effects of the dynamics in the process; (2) DET can model the possible accidental sequences, but at a large computational cost; (3) accurate modelling of sequences with low probabilities of occurrence can be achieved by the MCDET method.
\end{abstract}

Keywords-Monte Carlo simulation; dynamic event tree; Monte Carlo dynamic event tree; dynamics reliability.

\section{INTRODUCTION}

Probabilistic Safety Assessment (PSA) is performed by regulatory bodies to check the compliance of nuclear power plant design with regulatory requirements, and by industry for the identification of key vulnerabilities, so that the impact of operational changes on the operating plants can be informed by risk quantification [1-3].

Traditional PSA methods are, for example, Fault Trees (FTs) and Event Trees (ETs), that describe each accident sequence as a combination of success and failure events, accounting for the contribution of each component. Such PSA tools are currently widely used, but some limitations are acknowledged: the conservative assumptions that are made for the sake of PSA modelling simplification may lead to conservative results that, however, still do not assure coverage of the uncertainty therein (e.g. because of imprecise description of component aging and maintenance, binary modelling of components behavior, i.e., only faulty/safe states are considered, and neglecting dynamics of the system, i.e., the effect of order and timing of failure events on the accident progression) $[2,4]$.

To overcome some of these the limitations, dynamic reliability methods have been developed [5], such as Dynamic Event Tree (DET) [6, 7], the Continuous Cell-to-Cell-Mapping Technique (CCCMT) [8], Monte Carlo (MC) simulation [9], Markov/CCMT [10], Monte Carlo Dynamic Event Tree (MCDET) $[11,12]$. The methods of MC, DET and MCDET are the most popular ones.
This paper analyzes critically these latter methods by applying them to a classic level control dynamic system of literature [13-15] and to a realistic Emergency and Standby Power System (ESPS) [16, 17] drawing some conclusions on their strengths (i.e., accuracy) and weaknesses (i.e., computational demand and model complexity) for practical application.

The paper is organized as follows. The methods of MC, DET, MCDET are briefly recalled in Section 2. Section 3 presents and discusses the results of the application of the methods to the level control dynamic system. The ESPS results are presented in Section 4. Finally, conclusions are given in Section 5.

\section{THE DYNAMIC RELIABILITY METHODS CONSIDERED}

\section{A. Monte Carlo Simulation}

Monte Carlo (MC) simulation is a method that allows sampling the events that occur in an accident sequence from given probability distributions. For this, the MC simulation has two loops. The outer loop is iterated a number $N$ of times equal to the number $N$ of sequences to be simulated. This loop also allows for sampling values of process variables affected by epistemic uncertainty [13]. The inner loop allows sampling values of variables affected by aleatory uncertainty and simulates the occurrence of events along the sequence, up to the Mission Time $T_{M}$ [13]. A flowchart of the inner loop of a MC simulation is shown in Fig. 1.

Statistics of the $N$ sequences simulated by $\mathrm{MC}$, like the Failure Frequency (FF), Mean Time To Failure (MTTF), Mean Time To Repair (MTTF), Sensitivity Indexes (SI) can be estimated.

\section{B. Dynamic Event Tree}

A Dynamic Event Tree (DET) has a similar structure to its static counterpart, i.e. the ET, except that, in DET analysis, time is explicitly modelled so that the dynamic evolution of accidental sequences is modelled in a phenomenologically consistent manner [18]. A DET starts from an Initiating Event (IE) on the time axis and simulates the possible sequences which can develop from this IE, by branching at time points of the source branch of the tree. This allows for a wider and faster exploration of the failure domain of the system by simultaneously accounting for more than one sequence of events (contrary to what is done by MC simulation). There are two key issues to be addressed in DET construction: the selection of branching and stopping rules. The following rules are usually used in DET 
construction: (1) new branches are originated at discrete time points if the probability of the system to stay in the original state (i.e. to continue on the source branch) is smaller than a pre-defined branching probability threshold; (2) only one component can fail at each branching node; (3) when the generated branch probability is smaller than a pre-defined value, the generated branch is truncated and, thus, neglected in the following analysis. A flowchart of DET is shown in Fig. 2.

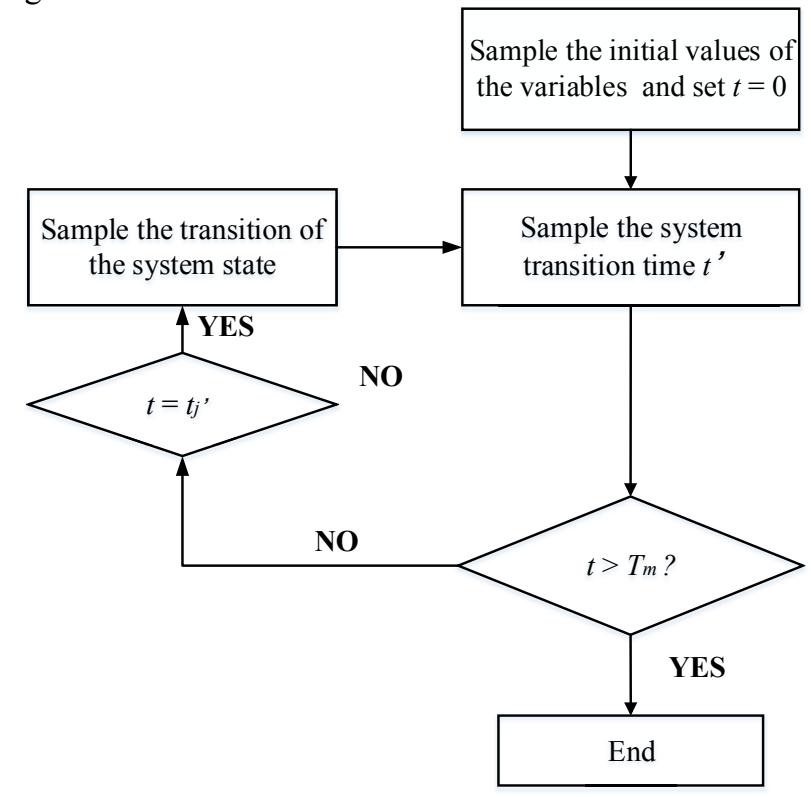

Figure 1. The flowchart of the inner loop of MC simulation

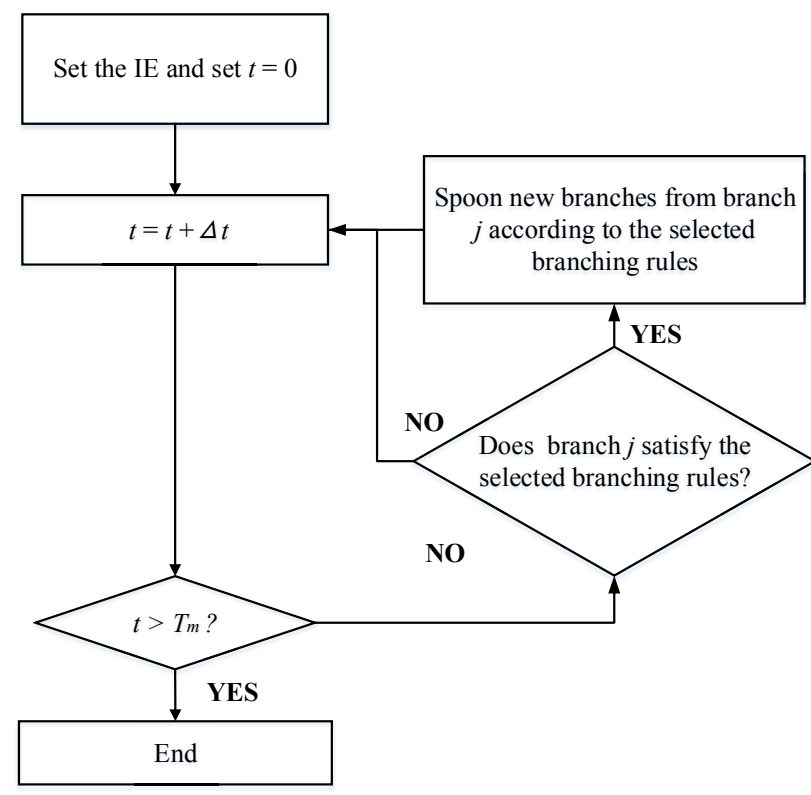

Figure 2. The flowchart of DET

\section{Monte Carlo Dynamic Event Tree}

Monte Carlo Dynamic Event Tree (MCDET) is a method that combines MC sampling and DET [19]. It is aimed at achieving an even more realistic modelling of system dynamics in the framework of probabilistic safety analyses. In MCDET models, continuous and random uncertainties are handled by MC simulation, while discrete and random uncertainties are treated by DET [11]. Like MC simulation, MCDET has two loops. The outer loop is iterated a number $N$ of times equal to the number $N$ of DETs to be simulated. In the inner loop, each DET starts from an IE and branches at time points along time $t$, up to the Mission Time $T_{M}$, that are sampled according to the MC simulation. As in $\mathrm{MC}$ simulation, epistemic uncertainties can also be considered by sampling from their probability distributions in the outer loop. A flowchart of the inner loop of a MCDET is shown in Fig. 3.

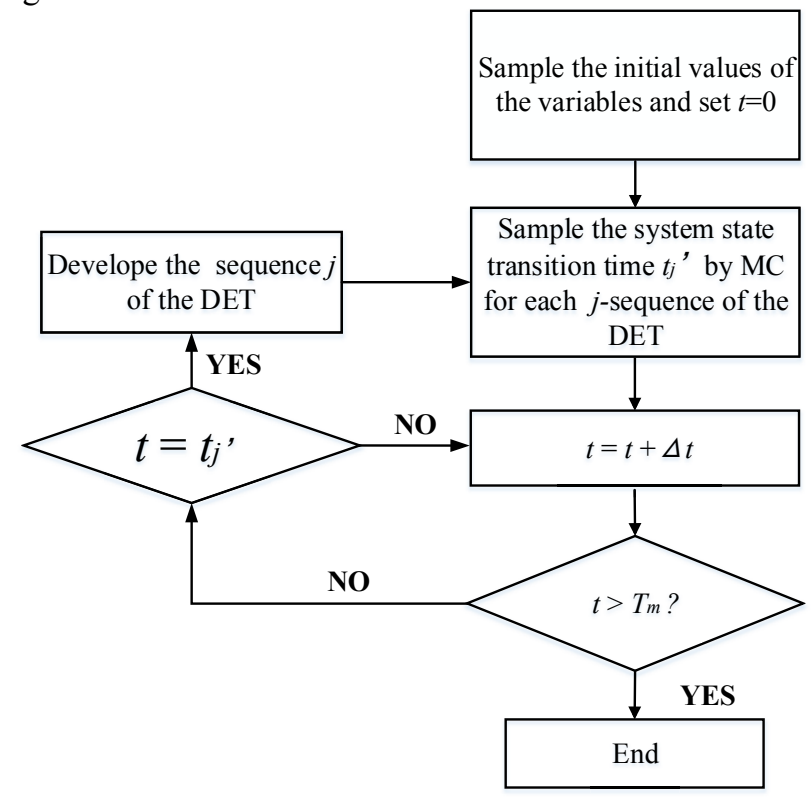

Figure 3. The flowchart of the inner loop of MCDET

\section{CASE Study 1: The LeVel Control DynamiC SYSTEM}

\section{A. Description of the System}

The system consists of two pumps and one valve that function so as to keep the fluid level of the tank within the interval $[6,8] \mathrm{m}$ by a control system, as shown in Fig. 3 . The scope is to keep the tank filled at a constant level $h$ equal to 7 $\mathrm{m}$, by injecting and discharging water at a rate $Q$ equal to 0.6 $\mathrm{m} / \mathrm{h}$ for pump 1 and the valve, respectively. In normal condition, pump 2 is in standby mode. In case the fluid exceeds any of the two thresholds of $6 \mathrm{~m}$ or $8 \mathrm{~m}$, the components states are changed by the control system as shown in Table I, where the rules to keep the level under control are listed. If some components are failed, some transitions might not be allowed and the control of the level might be lost, because the fluid might reach either "dry-out" level of $4 \mathrm{~m}$ or "over-flow" level of $10 \mathrm{~m}$. All the components can work in 4 possible states: safe-on and safe-off (i.e., normal condition), and stuck-on and stuck-off (i.e., failed condition). Different kinds of transition rates can be considered for describing the probabilities of transitions of these components among the possible states: constant and 
independent on the system state (Table II) or increasing with time and dependent on the system state (Table III). These assumptions lead to considering different probabilistic models: static reliability methods in the former case, dynamic methods (MC, DET and MCDET) in the latter cases.

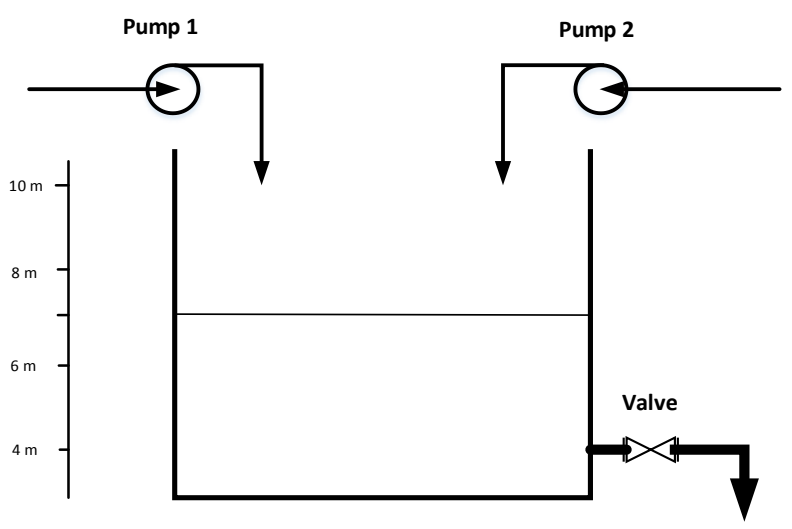

Figure 4. Diagram of the level control dynamic system

TABLE I. Control Rules of THE LeVel Control Dynamic System

\begin{tabular}{|c|c|c|c|}
\hline & Pump 1 & Pump 2 & Valve \\
\hline Fuild level $>8 \mathrm{~m}$ & Off & Off & On \\
\hline Fuild level $<6 \mathrm{~m}$ & On & On & Off \\
\hline
\end{tabular}

TABLE II. FAILURE RATES FOR THE STATIC PSAANALYSIS

\begin{tabular}{|l|c|c|}
\hline & $\boldsymbol{\lambda}_{\text {on }}$ & $\boldsymbol{\lambda}_{\text {off }}$ \\
\hline Pump 1 & $4.566 \mathrm{e}-3$ & $4.566 \mathrm{e}-3$ \\
\hline Pump 2 & $5.714 \mathrm{e}-3$ & $5.714 \mathrm{e}-3$ \\
\hline Valve & $3.125 \mathrm{e}-3$ & $3.125 \mathrm{e}-3$ \\
\hline
\end{tabular}

\section{B. Static PSA Analysis}

The failure rate $\lambda$ of each component of the system is considered to be constant and independent on the system state, $\lambda=\lambda_{\text {on }}=\lambda_{\text {off }}$ (See (Table II). Moreover, the probability that a component fails stuck-on/ stuck-off before time $t$ is assumed equal to:

$$
p_{\text {stuck-on }}(t)=p_{\text {stuck-off }}(t)=\frac{1}{2}[1-\exp (-\lambda t)]
$$

The reliability of each component at time $t$ is assumed equal to:

$$
R(t)=\exp (-\lambda t)
$$

The probability of the top event ("over-flow" and "dry-out"), under the rare event approximation, equals to:

$$
F(t)=\sum_{r=1}^{m} \prod_{x_{b} \in E_{r}} q_{b}(t)
$$

where:

$m \quad$ is the number of Minimal Cut Sets (MCSs) causing the top event ("over-flow" and "dry-out");

$E_{r} \quad$ is the $r^{\text {th }} \mathrm{MCS}$ causing the top event;

$x_{b} \quad$ is the $b^{\text {th }}$ basic event in the $r^{\text {th }} \operatorname{MCS} E_{r}$;

$q_{b}(t)$ is the probability of occurrence of the basic event $x_{b}$ before time $t$.

Table III lists the $\mathrm{m}=6 \mathrm{MCS}$ for the "over-flow" and "dry-out" top events. The probabilities of the top events to occur within $T_{m}=30 \mathrm{~h}$ are 0.0110 and 0.000226 respectively, and 0.4080 and 0.0836 for $T_{m}=500 \mathrm{~h}$. The plots of $F(t)$ are shown in Fig. 5.

\begin{tabular}{|c|c|}
\hline Top event & Minimal Cut Sets (MCSs) \\
\hline \multirow{5}{*}{$\begin{array}{l}\text { "over-flow" } \\
\text { (5 MCSs) }\end{array}$} & Pump $1_{\text {stuck on }}$, Pump $2_{\text {stuck off }}$, Valve $_{\text {stuck off }}$ \\
\hline & Pump $1_{\text {stuck off }}$ Pump $2_{\text {stuck on }}$, Valve $_{\text {stuck off }}$ \\
\hline & Pump1 $1_{\text {safe }}$, Pump $2_{\text {stuck on }}$, Valve stuck off \\
\hline & Pump $1_{\text {stuck on }}$, Pump $2_{\text {safe }}$, Valve $_{\text {stuck off }}$ \\
\hline & Pump $1_{\text {stuck on }}$, Pump $2_{\text {stuck on }}$ \\
\hline $\begin{array}{l}\text { "dry-out" } \\
\text { (1 MCS) }\end{array}$ & Pump $1_{\text {stuck off }}$, Pump $2_{\text {stuck off }}, \quad$ Valve stuck on \\
\hline
\end{tabular}

TABLE III. Minimal Cut Sets of THE FaILURE OF THE LeVel CONTROL DYNAMIC SYSTEM [15]

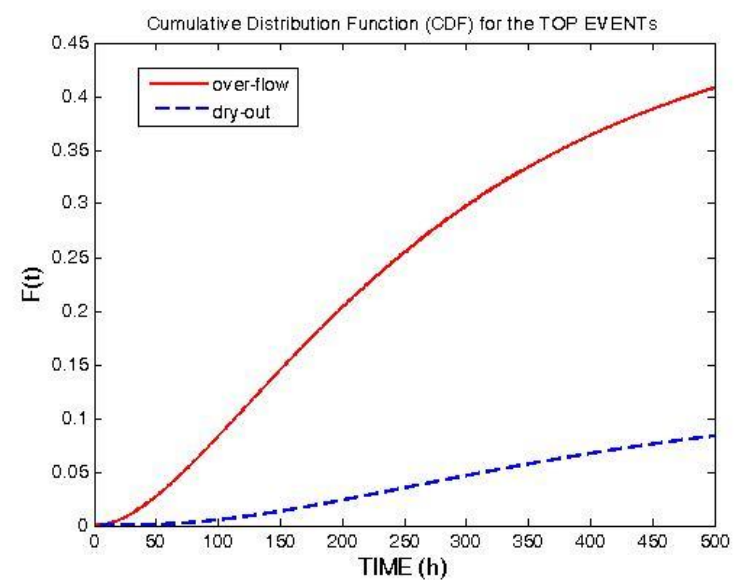

Figure 5. Cumulative Distribution Function (CDF) for the top events "over-flow" and "dry-out"

\section{Dynamic PSA Analysis}

The demand of reality in modeling the behavior of components and systems calls for transition rates that account for aging (i.e., they increase with time) and that depend on the system state. For the impact of aging on the transition rates, we assume the following linear form [20]:

$$
\lambda(t)=\lambda_{0}+k t
$$

where:

$\lambda(t)$ is the transition rate at time $t$,

$\lambda_{0}$ is the design transition rate, (i.e. the value at time $t=0$ ),

$k \quad$ is the aging factor

The transition rates dependences on the system state are given in Table IV.

Under these circumstances, we adopt dynamic methods for reliability modelling. To benchmark the different 
methods and compare with the static method of section 3.2, four different cases are considered:

1) no aging and no transition rates dependence on the system state

2) slow aging and no transition rates dependence on the system state

3) fast aging and no transition rates dependence on the system state

4) no aging and transition rates dependence on the system state

Control rules of the level control dynamic system listed in Table I hold for all the four above mentioned cases. For each case, MC, DET and MCDET models are constructed for $T_{m}=30 \mathrm{~h}$. The time step $\Delta t$ considered is set equal to $0.1 \mathrm{~h}$ for MC and MCDET, whereas for the DET model, is set equal to $0.66 \mathrm{~h}$. The DET branching probability threshold and truncation probability threshold, aimed at reducing the branching explosion, are set equal to 0.999 and $1 \times 10^{-9}$ (i.e., branches with lower probability are neglected), respectively.

All the dynamic models have been run on a single node of the super-computing center of the University of Science and Technology of China (USTC) (Intel Xeon E5620, 4 CPU, 2.4GHz, 16GB)

TABLE IV. Transition Rates That Depend on the System State

\begin{tabular}{|l|c|c|c|c|}
\hline & $\lambda_{\text {safe }- \text { off } \rightarrow \text { stuck-off }}$ & $\lambda_{\text {safe }- \text { off } \rightarrow \text { stuck-on }}$ & $\lambda_{\text {safe-on } \rightarrow \text { stuck-off }}$ & $\boldsymbol{\lambda}_{\text {safe-on } \rightarrow \text { stuck-on }}$ \\
\hline Pump 1 & $4.566 \mathrm{e}-1$ & $4.566 \mathrm{e}-2$ & $4.566 \mathrm{e}-3$ & $4.566 \mathrm{e}-3$ \\
\hline Pump 2 & $5.714 \mathrm{e}-3$ & $5.714 \mathrm{e}-3$ & $5.714 \mathrm{e}-1$ & $5.714 \mathrm{e}-2$ \\
\hline Valve & $3.125 \mathrm{e}-1$ & $3.125 \mathrm{e}-2$ & $3.125 \mathrm{e}-3$ & $3.125 \mathrm{e}-3$ \\
\hline
\end{tabular}

\section{Results and Discussions}

In Fig. 6, the plots of $F(t)$ obtained with the static PSA analysis of Section 3.2 (continuous line) are compared with those of the dynamic PSA analysis of Section 3.3 (crosses, left and right triangles for DET, MC and MCDET, respectively), for the case of no aging and no transition rates dependence on the system state. It can be seen that the static PSA model overestimates both failure modes with respect to the dynamic models, at any time. This is mainly due to the assumption made with the static modelling, for which the system fails as soon as any of its MCS occurs; however, the real dynamics of the system does not imply the sudden system failure when a MCS occurs. For example if one pump fails stuck-on and the valve stuck-off at any time $t$, the system exceeds the level thresholds only several hours later due to the actual discharging/injecting rate $\mathrm{Q}$ of the pumps and valve, that is, instead, neglected in the static PSA model.

On one hand, such assumption in static PSA causes the probabilities of the top events to be greater than the actual ones. On the other hand, the capability of dynamic probabilistic methods to properly account for the dynamics into the quantification become evident.

Focusing on the dynamic methods, Fig. 6 (right) shows that the estimate provided by the MCDET is smoother than that provided by the $\mathrm{MC}$ simulation, because the former (by exploring simultaneously more than one branch of the possible developing scenarios) allows collecting more evidence of system failure than the latter with the same computational effort (as we shall see in what follows).

The capability of dynamic methods to catch the aging effects is shown in Fig. 7 and Fig. 8 for $T_{m}=30 \mathrm{~h}$ and $T_{m}=$ $500 \mathrm{~h}$, respectively. It can be seen that, at the early stage of the system life, the impact of the components aging on the result is negligible (crosses, left and right triangles are almost overlapped), because the change of the transition rates caused by the aging factors is limited (see Eq (4)). On the other hand, as long as the system degrades, the aging effect is predominant and the transition rates values increase, and the impact becomes considerable already after 100 hours for $k=1 \mathrm{e}-5$ (left triangles).

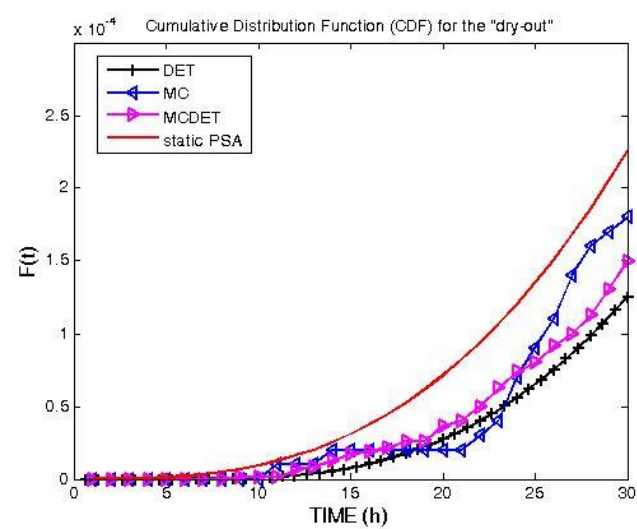

Figure 6. Cumulative Probability Distribution for "over-flow" (left) and "dry-out" (right) with $T_{m}=30 \mathrm{~h}$ and N $=100000$, for the case of no aging and no transition rates dependence on the system state 

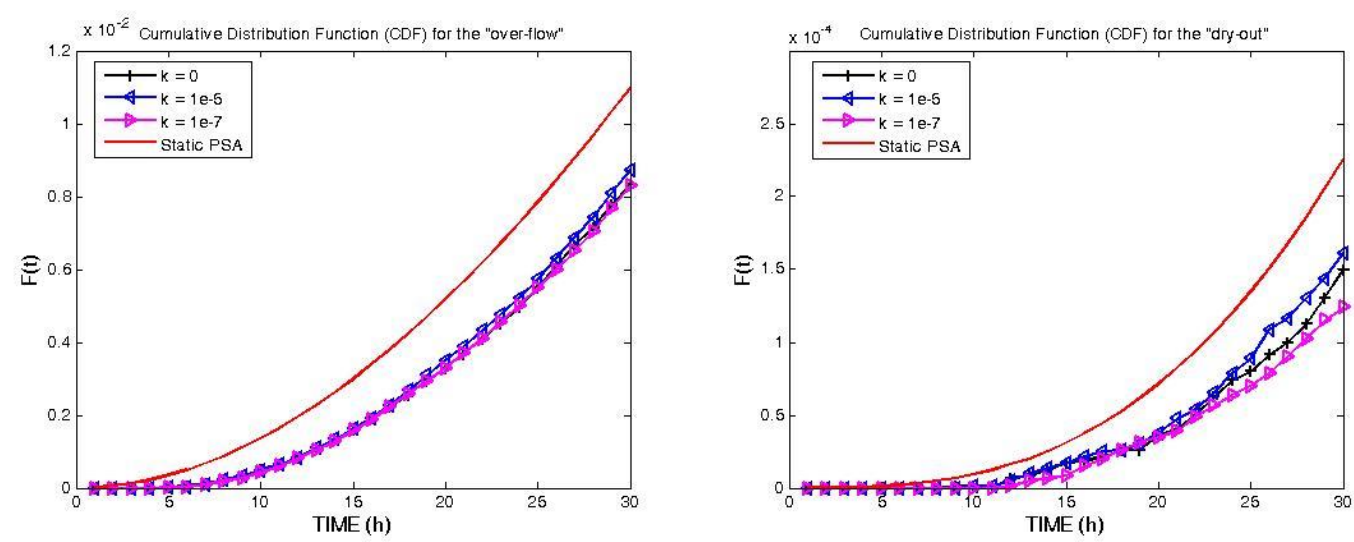

Figure 7. Cumulative Probability Distribution for "over-flow" (left) and "dry-out" (right) with $T_{m}=30 \mathrm{~h}$ and N=10000, for the case of aging and no transition rates dependence on the system state
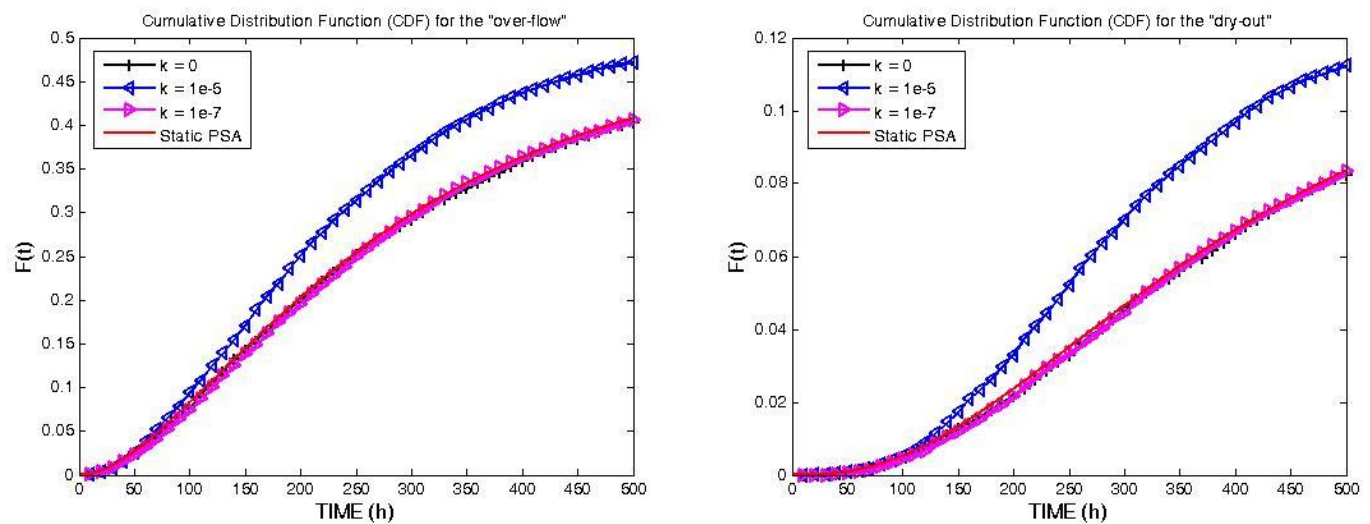

Figure 8. Cumulative Probability Distribution for "over-flow" (left) and "dry-out" (right) with $T_{m}=500 \mathrm{~h}$ and N=10000, for the case of aging and no transition rates dependence on the system state

The capability of dynamic methods to catch the dependence of the components transition rates on the system state (Table III and case 4) is shown in Fig. 9 and Fig. 10. It can be seen that the probability of the system failure in the early stage is much larger than the ones shown in Fig. 6, especially for the probabilities of "dry-out". It is worthy to note that as long as time increases, the "over-flow" and

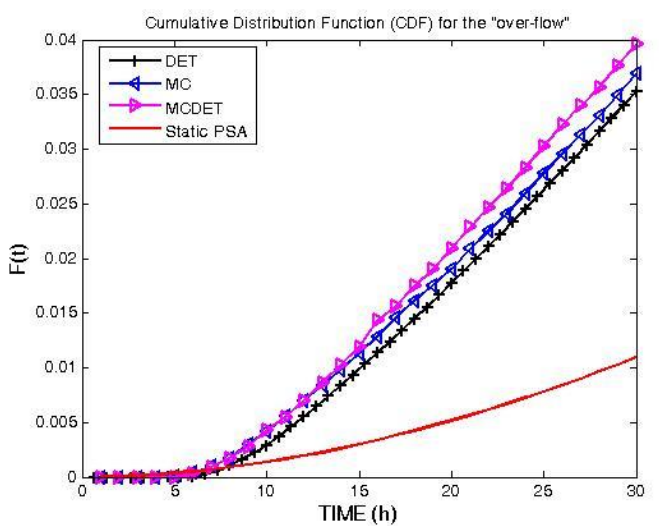

"dry-out" probabilities reach the estimated values of Fig. 6 at about $370 \mathrm{~h}$ and $470 \mathrm{~h}$, respectively. This is due to the fact that the considered level control system is a non-coherent system. Although transition rates increase with respect to the base case of no aging and no dependence on the system state, the number of component contributions that lead the system into any of the two top failure modes decreases.

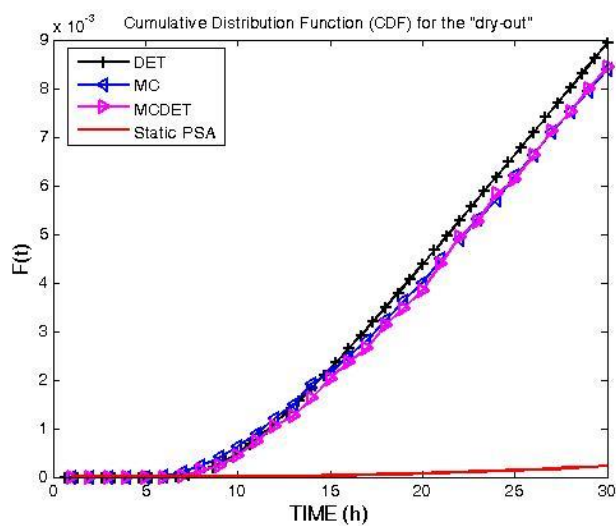

Figure 9. Cumulative Probability Distribution for "over-flow" (left) and "dry-out" (right) with $T_{m}=30 \mathrm{~h}$ and N=100000, for the case of no aging and transition rates dependence on the system state 

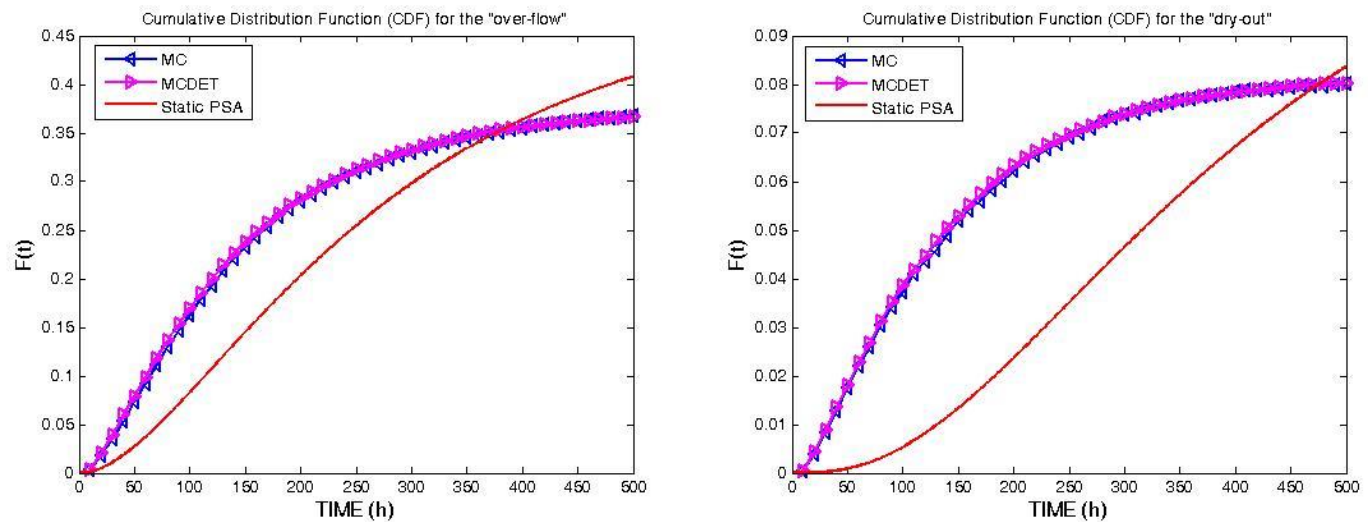

Figure 10. Cumulative Probability Distribution for "over-flow" (left) and "dry-out" (right) with $T_{m}=500 \mathrm{~h}$ and $\mathrm{N}=10000$, for the case of no aging and transition rates dependence on the system state

TABLE V. COMPUTATIONAL EFFICIENCY For DET, MC AND MCDET MODELS

\begin{tabular}{|c|c|c|c|c|c|c|c|}
\hline Case & Model & $\begin{array}{l}\text { Number of } \\
\text { Cycles, } N\end{array}$ & $\begin{array}{c}\text { Computational } \\
\text { cost }[\mathrm{s}]\end{array}$ & $\begin{array}{l}\text { Number of } \\
\text { "over-flow" }\end{array}$ & $\begin{array}{l}\text { Number of } \\
\text { "dry-out" }\end{array}$ & $\begin{array}{l}\text { Expected time to } \\
\text { "over-flow" [s] }\end{array}$ & $\begin{array}{l}\text { Expected time } \\
\text { to "dry-out" [s] }\end{array}$ \\
\hline \multirow{5}{*}{ Case 1} & $\operatorname{DET}\left(T_{m}=30 \mathrm{~h}\right)$ & - & 16004 & 157784 & 52914 & - & - \\
\hline & $\operatorname{MC}\left(T_{m}=30 \mathrm{~h}\right)$ & 300000 & 656272 & 2643 & 36 & 248 & 18229 \\
\hline & $\operatorname{MCDET}\left(T_{m}=30 \mathrm{~h}\right)$ & 100000 & 219123 & 3496 & 124 & 63 & 1767 \\
\hline & $\mathrm{MC}\left(T_{m}=500\right)$ & 10000 & 30668 & 3996 & 826 & 7.67 & 37.13 \\
\hline & $\operatorname{MCDET}\left(T_{m}=500\right)$ & 3000 & 14606 & 6851 & 1977 & 2.13 & 7.39 \\
\hline \multirow{5}{*}{ Case 2} & $\operatorname{DET}\left(T_{m}=30 \mathrm{~h}\right)$ & - & 9669 & 157784 & 52914 & - & - \\
\hline & $\mathrm{MC}\left(T_{m}=30 \mathrm{~h}\right)$ & 300000 & 638364 & 2494 & 39 & 256 & 16368 \\
\hline & $\operatorname{MCDET}\left(T_{m}=30 \mathrm{~h}\right)$ & 100000 & 241849 & 3479 & 100 & 70 & 2419 \\
\hline & $\mathrm{MC}\left(T_{m}=500\right)$ & 10000 & 21322 & 4124 & 790 & 5.17 & 26.99 \\
\hline & $\operatorname{MCDET}\left(T_{m}=500\right)$ & 3000 & 14410 & 6892 & 1999 & 2.09 & 7.21 \\
\hline \multirow{5}{*}{ Case 3} & $\operatorname{DET}\left(T_{m}=30 \mathrm{~h}\right)$ & - & 9524 & 157784 & 52914 & - & - \\
\hline & $\operatorname{MC}\left(T_{m}=30 \mathrm{~h}\right)$ & 300000 & 657328 & 2701 & 39 & 243 & 16855 \\
\hline & $\operatorname{MCDET}\left(T_{m}=30 \mathrm{~h}\right)$ & 100000 & 246248 & 3707 & 131 & 66 & 1880 \\
\hline & $\mathrm{MC}\left(T_{m}=500\right)$ & 10000 & 34685 & 4745 & 1099 & 7.31 & 31.56 \\
\hline & $\operatorname{MCDET}\left(T_{m}=500\right)$ & 3000 & 16208 & 8414 & 2697 & 1.93 & 6.01 \\
\hline \multirow{5}{*}{ Case 4} & $\operatorname{DET}\left(T_{m}=30 \mathrm{~h}\right)$ & - & 8523 & 127482 & 45554 & - & - \\
\hline & $\operatorname{MC}\left(T_{m}=30 \mathrm{~h}\right)$ & 300000 & 308595 & 3729 & 929 & 83 & 332 \\
\hline & $\operatorname{MCDET}\left(T_{m}=30 \mathrm{~h}\right)$ & 20000 & 33189 & 10795 & 3333 & 3.07 & 9.99 \\
\hline & $\mathrm{MC}\left(T_{m}=500\right)$ & 30000 & 71527 & 11014 & 2024 & 6.49 & 29.75 \\
\hline & $\operatorname{MCDET}\left(T_{m}=500\right)$ & 10000 & 42566 & 2956 & 9856 & 1.51 & 4.59 \\
\hline
\end{tabular}

Although dynamic methods (MC, DET and MCDET) have shown superior capabilities with respect to static PSA methods, the computational cost may be large. The computational cost, indeed, depends on the length of the discrete time step that is simulated, the length of the mission time $T_{m}$ to be simulated, the branching probability thresholds that is set (only for DET) and the selected number of repeated sampling cycles $N$ (only for MC and MCDET). Fig. 11 shows the computational costs of the MC, DET and MCDET models for the base case of no aging and no 
transition rates dependence on the system state as a function of $T_{m}$. The computational cost of DET model increases exponentially as the $T_{m}$ increases, while the computational costs of MC and MCDET models increase approximately linearly with $T_{m}$. So DET model is only applicable when a limited $T_{m}$ is foreseen to be modelled. For long $T_{m}$, MC and MCDET models seem more appropriate than DET.

A more detailed analysis and comparison among DET, $\mathrm{MC}$ and MCDET is presented in Table $\mathrm{V}$, that lists the computational efficiency of the selected dynamic methods when applied to the four cases 1), 2), 3) and 4) illustrated in section 3.3. For the purpose of comparison, we define computational efficiency as the capability of a method to collect evidences of system failure (i.e., number of "over-flow" and "dry-out") with a given computational effort (i.e., number of $\mathrm{MC}$ cycles $(N)$, computational cost [s], expected time to "over-flow" [s] and to "dry-out" [s]). The results show that the MCDET method is much more effective to collect the evidences of "over-flow" and "dry-out" than MC simulation at same $T_{m}$, because the sequences with low probability of occurrence are not discarded. For example, when no aging and no dependence of the transition rates on the system state (case 1 ) is simulated and $T_{m}$ is equal to 30 $\mathrm{h}$, the expected times for simulating one "over-flow" with $\mathrm{MC}$ and MCDET are $248 \mathrm{~s}$ and $63 \mathrm{~s}$, respectively, and the expected times to "dry-out" are $18229 \mathrm{~s}$ and $1767 \mathrm{~s}$, respectively.

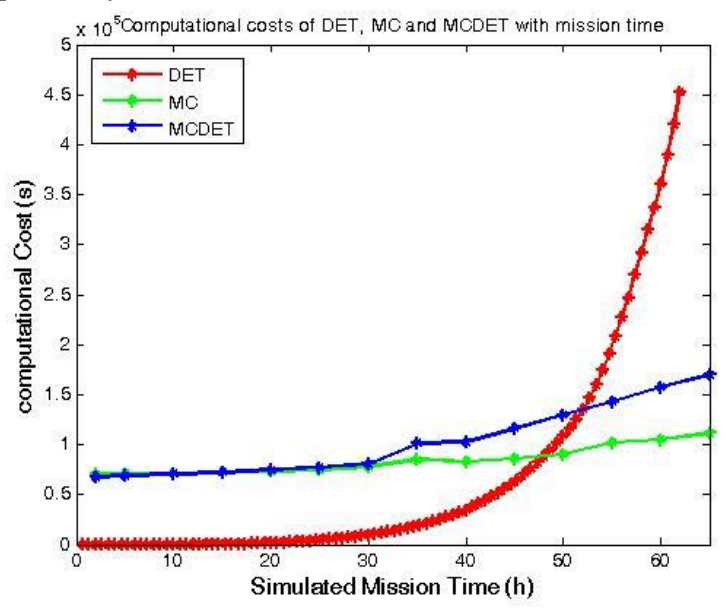

Figure 11. Computational cost of DET, MC and MCDET as a function of the mission time $T_{m}$

\section{CASe Study 2: The EMERgency And Standby Power SYSTEM (ESPS)}

\section{A. Description of the System}

Emergency and standby power systems (ESPS) are designed to provide a plant alternative source of power when the normal source of power, the Utility Input Power (UIP), fails. A sketch of the system is shown in Fig. 12 [16, 17]. The power is normally supplied by the UIP while two generators (G1 and G2) are kept in cold standby mode, with probability of failure on demand $p_{s}$ equal to 0.015 . A synchronized bypass and a static transfer switch (STS) protect the critical load in the event of inverter failure. If voltage is lost at the Critical Load Bus (CLB), STS reestablishes voltage in less than one-quarter of a cycle. If the power fails at bus A, the battery can supply the power for $4 \mathrm{~h}$.

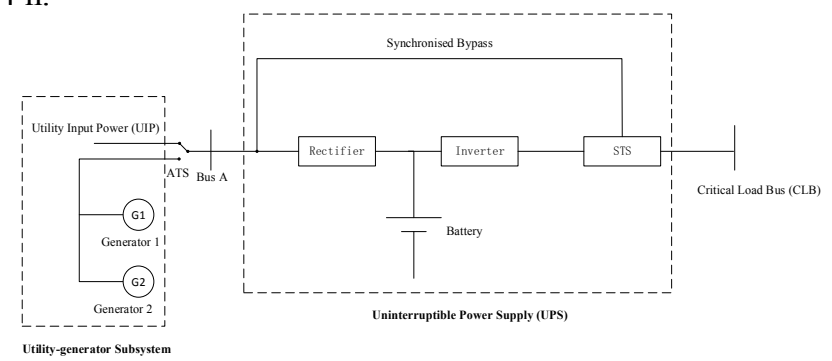

Figure 12. Diagram of Emergency and Standby Power Systems

Table VI shows the reliability data for all the components of the ESPS system that are considered repairable. It is important to notice that only one generator can be taken out for maintenance and that if a generator fails while another is on maintenance, the maintenance on the second generator would be accelerated by a factor of $\alpha$, setting $\alpha=2$ in this case. The fault tree for the top event "power loss at the Critical Load Bus (CLB)" is shown in Fig. 13.

TABLE VI. RELIABILITY DATA FOR VARIOUS COMPONENTS OF THE ESPS SYSTEM

\begin{tabular}{lcc}
\hline \hline Equipment/Supply & $\boldsymbol{\lambda}(\mathbf{f} / \mathbf{y})$ & $\mathbf{R}(\mathbf{h} / \mathbf{f})$ \\
\hline Utility Input Power & 0.53700 & 5.66 \\
Generator (G1/G2) (per hour of use) & 0.00536 & 478.00 \\
Inverter & 1.25400 & 107.00 \\
Rectifier & 0.03800 & 39.00 \\
Automatic Transfer Switch (ATS) & 0.00600 & 5.00 \\
Static Transfer Switch (STS) & 0.08760 & 24.00 \\
Battery & 0.03130 & 24.00 \\
\hline \hline Equipment Maintenance & Frequency (/y) & Duration (h) \\
\hline Generator (G1/G2) & 1.00 & 10.00 \\
Uninterruptible Power Supply (UPS) & 1.00 & 4.00 \\
\hline \hline
\end{tabular}

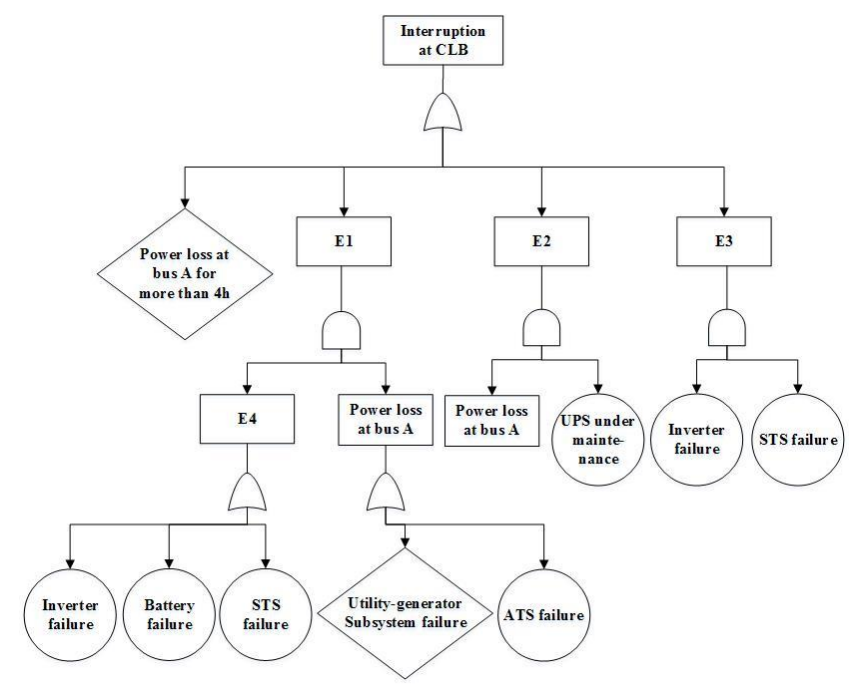

Figure 13. Fault tree of "power loss at the Critical Load Bus (CLB)"

The reliability analysis of the ESPS system is made by the MC method, the Markov method and minimal cut set 
method, respectively in reference [16] and [17]. In this work, we benchmark the results with those that we obtain with $\mathrm{MC}$ and MCDET models.

\section{B. Dynamic PSA Analysis}

MC and MCDET models are built for the reliability analysis of the ESPS system. These would allow considering the dependencies between components (i.e., accidental maintenance on generators, when at least one of the two is out of order). The time discretization considered for the simulation is set equal to $0.1 \mathrm{~h}$. The only difference between the two models is that only one sequence of events can be simulated with one cycle of MC model, whereas for MCDET, that same sequence of event may branch out into several branches at the selected branching nodes of the DET. Two models are run on the single node of the super-computing center of USTC (Intel Xeon E5620, 4 CPU, 2.4GHz, 16GB).

\section{Results and Discussions}

MC and MCDET models are used to compute the reliability parameters for the ESPS (listed in Table VII) to be compared with those obtained with Markov methods [17]: frequency $\left(f_{p}\right)$ and duration $\left(r_{p}\right)$ of failure of the utility-generator subsystem, frequency $\left(f_{A}\right)$ and duration $\left(r_{A}\right)$ of power loss at bus $A$, and frequency $\left(f_{C L B}\right)$ and duration $\left(r_{C L B}\right)$ of power loss at CLB estimated confidence intervals (i.e., estimated values \pm standard deviation) are all bounding the values calculated in [17], confirming the capability of $\mathrm{MC}$ and MCDET of effectively modelling the system dynamics.

The computational efficiency in capturing the evidence of failure for each developed dynamic model is shown in Table VIII. The same conclusion of Section 3.4 can be drawn from this case study: the MCDET method is more effective to collect evidences of failure with respect to $\mathrm{MC}$ simulation. For example, the MC model with 50000 cycles models cut-set E1 three times whereas cut-set E2 is modelled zero times within mission time $T_{m}=1 y$, while the MCDET model, with the same number of cycles and $T_{m}$, models cut-set E1 42 times and cut-set E2 only 2 times. The probability distributions of duration of power loss at the CLB obtained by the two dynamic models are displayed in Fig. 14.

TABLE VII. RELIABILITY PARAMETERS OBTAINED FROM MC AND MCDET MODELS

\begin{tabular}{|c|c|c|c|c|c|}
\hline \multirow{2}{*}{} & \multirow{2}{*}{$\begin{array}{c}\text { Estimated } \\
\text { value in [17] }\end{array}$} & \multicolumn{2}{|c|}{ MC (50000 cycles) } & \multicolumn{2}{c|}{ MCDET 50000 cycles) } \\
\cline { 3 - 6 } & & $\begin{array}{c}\text { Estimated } \\
\text { value }\end{array}$ & $\begin{array}{c}\text { Confidence interval } \\
\text { [Estimated value } \pm \\
\text { standard deviation] }\end{array}$ & $\begin{array}{c}\text { Estimated } \\
\text { value }\end{array}$ & $\begin{array}{c}\text { Confidence interval } \\
\text { [Estimated value } \pm \\
\text { standard deviation] }\end{array}$ \\
\hline$f_{p}$ & 0.00157 & 0.00184 & {$[0.00152,0.00216]$} & 0.00184 & {$[0.00158,0.00211]$} \\
\hline$r_{p}$ & 5.4532 & 5.5152 & {$[4.5038,6.5266]$} & 5.5072 & {$[5.3368,5.6776]$} \\
\hline$f_{A}$ & 0.00757 & 0.00798 & {$[0.00733,0.00863]$} & 0.00781 & {$[0.00718,0.00843]$} \\
\hline$r_{A}$ & 5.0938 & 5.0005 & {$[4.9656,5.0354]$} & 5.3407 & {$[5.1873,5.4941]$} \\
\hline$f_{C L B}$ & 0.00523 & 0.00540 & {$[0.00486,0.00594]$} & 0.00593 & {$[0.00537,0.00648]$} \\
\hline$r_{C L B}$ & 9.648 & 11.5402 & {$[10.3767,12.7037]$} & 9.4789 & {$[9.1177,9.8402]$} \\
\hline
\end{tabular}

TABLE VIII. STATISTICS OF COMPUTATIONAL EFFICIENCIES OF CAPTURING THE EVIDENCE OF FAILURE FOR EACH MOdEL

\begin{tabular}{|l|c|c|c|c|}
\hline \multirow{2}{*}{ Events } & \multicolumn{2}{|c|}{ MC (50000 cycles) } & \multicolumn{2}{c|}{ MCDET (50000 cycles) } \\
\cline { 2 - 5 } & $\begin{array}{l}\text { Number } \\
\text { evidences }\end{array}$ & $\begin{array}{l}\text { Expected time for } \\
\text { capturing an evidence }\end{array}$ & $\begin{array}{l}\text { Number of } \\
\text { evidences }\end{array}$ & $\begin{array}{l}\text { Expected time for } \\
\text { capturing an evidence }\end{array}$ \\
\hline Failure of utility-generator subsystem & 102 & 747 & 2770 & 73 \\
\hline Failure of ATS & 307 & 248 & 550 & 370 \\
\hline Failure of power supply over 4h & 47 & 1587 & 1330 & 153 \\
\hline Failure of ATS over 4h & 141 & 540 & 261 & 780 \\
\hline Cut-set E1 & 3 & 25392 & 42 & 4845 \\
\hline Cut-set E2 & 0 & - & 2 & 101739 \\
\hline Cut-set E3 & 78 & 977 & 161 & 1264 \\
\hline Power loss at CLB & 276 & 276 & 1796 & 113 \\
\hline
\end{tabular}

\section{CONCLUSION}

This paper analyzes dynamic methods for PSA by application to two systems. The methods considered (MC, DET and MCDET) are shown to overcome the classic PSA methods (FT/ET), for systems where dynamic factors like aging and dependence among components play a significant role. They, indeed, allow accounting for the variation of failure rates depending on system state, and the order and timing of failure events along an accidental scenario. As a 
matter of fact, as shown with respect to the level control system and ESPS system case studies, dynamic methods are more flexible to incorporate various effects of the dynamics of the process, compared with static PSA methods. A comparison of the dynamic PSA methods implemented shows that the computational costs of the DET method are large when long mission times are to be simulated, and that MCDET is more effective to simulate failure sequences of events than MC method with the same computational effort.

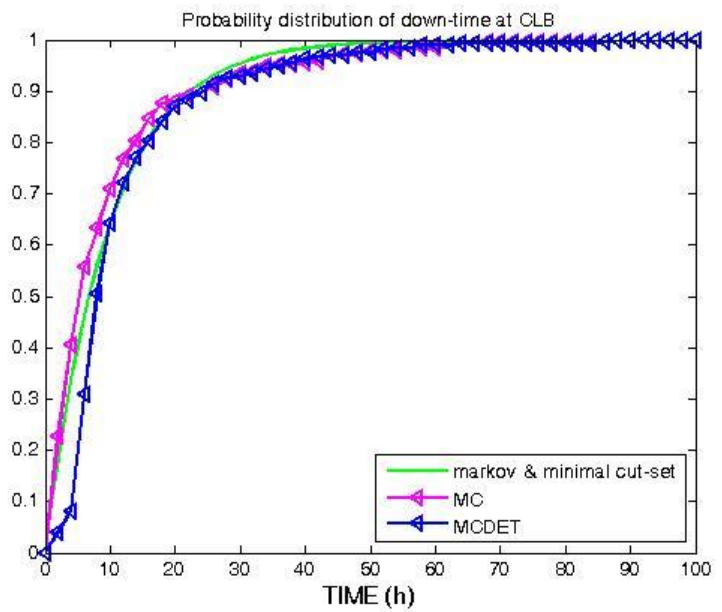

Figure 14. Probability distributions of duration of power loss at the CLB

\section{ACKNOWLEDGEMENT}

The numerical calculations in this paper have been done on the supercomputing system in the Supercomputing Center of University of Science and Technology of China. We further thank the great help from other members of LASAR Team in this research.

\section{REFERENCES}

[1] IAEA. Applications of probabilistic safety Assessment (PSA) for nuclear power plants. IAEA-TECDOC-1200, 2001

[2] F. Di Maio, M. Vagnoli, and E. Zio, Transient identification by clustering based on integrated deterministic and probabilistic safety analysis outcomes. Annals of Nuclear Energy, vol. 87, Part 2: pp. 217-227. 2016

[3] F. Di Maio, P. Secchi, S. Vantini, and E. Zio, Fuzzy C-means clustering of signal functional principal components for post-processing dynamic scenarios of a nuclear power plant digital instrumentation and control system. IEEE Transactions on Reliability, Vol. 60 (2), pp. 415-425. June 2011.
[4] F. Di Maio, S. Baronchelli, E. Zio, A computational framework for prime implicants identification in noncoherent dynamic systems. risk analysis. Vol. 35(1): pp. 142-156. 2015.

[5] T. Aldemir, A survey of dynamic methodologies for probabilistic safety assessment of nuclear power plants. Annals of Nuclear Energy. Vol. 52: pp. 113-124. 2013.

[6] E. Hofer, M. Kloos and B. Krzykacz-Hausmann, Dynamic event trees for probabilistic safety analysis. GRS, Garsching, Germany, 2004.

[7] C. Acosta and N.O. Siu, Dynamic event tree analysis method (DETAM) for accident sequence analysis. Massachusetts Institute of Technology, 1991.

[8] B. Tombuyses and T. Aldemir, Continuous cell-to-cell mapping. Journal of sound and Vibration. Vol. 202(3): pp. 395-415. 1997.

[9] E. Zio, The Monte Carlo simulatn method for system reliability and risk analysis. Springer. 2013

[10] I.B. Gomes, P.F. Melo, and P.L. Saldanha, A cell-to-cell Markovian model for the reliability of a digital control system of a steam generator. UFRJ/COPPE/Programa de Engenharia Nuclear, 2013.

[11] M. Kloos and J. Peschke, MCDET: a probabilistic dynamics method combining monte carlo simulation with the discrete dynamic event tree approach. Nuclear science and engineering. Vol. 153(2): pp. 137-156. 2006

[12] M. Sonnenkalb, J. Peschke and M. Kloos, MCDET and MELCOR-an example of a stochastic module coupled with an integral code for PSA Level 2. International Workshop on Level 2 PSA and Severe Accident Management. Köln, Germany. 2004.

[13] D.R. Karanki, V.N. Dang, and M.T. MacMillan, Uncertainty propagation in dynamic event trees - initial results for a modified tank problem. Probabilistic Safety Assessment and Management PSAM 12, Honolulu, Hawaii. 2014.

[14] F. Di Maio, M. Stasi and E. Zio, Identification of faults in a level control dynamic system. ANS NPIC HMIT 2009 Topical Meeting Nuclear Plant Instrumentation, Controls, and Human Machine Interface Technology, Knoxville, Tennessee. pp. 1228-1239. 2009.

[15] M. Marseguerra and E. Zio, Monte Carlo approach to PSA for dynamic process systems. Reliability Engineering and System safety. Vol. 52. pp. 227-241. 1996.

[16] C. Singh and J. Mitra, Monte Carlo simulation for reliability analysis of emergency and standby power systems. In Industry Applications Conference. Vol. 3. Pp. 2290-2295. 1995,

[17] C. Singh, N. Gubbala, and N. Gubbala, Reliability analysis of electric supply including standby generators and an uninterruptible power supply system. IEEE transactions on industry applications. 130(5): pp. 1298-1302. 994

[18] Nuclear Regulatory Commission. Severe accident risks: an assessment for five US nuclear power plants. No. NUREG--1150-vol. 3. Nuclear Regulatory Commission, 1991.

[19] E. Hofer, M. Kloos, and B. Krzykacz. Methodenentwicklung zur simulativen Behandlung der Stochastik in probabilistischen Sicherheitsanalysen der Stufe 2. Garching: GRS-A-2997, Gesellschaft für Anlagen-und Reaktorsicherheit, Germany.2001.

[20] D. Kančev and M. Čepin. Ageing within PSA: development of an analytical unavailability model and its application. European Nuclear Young Generation Forum, Prague. 2011. 